\section{JTI}

JOURNAL OF

TRAUMA AND INJURY

Received: August 24, 2019

Revised: October 28, 2019

Accepted: November 12, 2019

\section{Correspondence to}

Seong Ho Choi, M.D.

Department of Surgery, Ulsan University Hospital, University of Ulsan College of

Medicine, 881 Bangeojinsunhwando-ro,

Dong-gu, Ulsan 44033, Korea

Tel: $+82-52-250-7118$

Fax: +82-52-250-8150

E-mail:0734494@uuh.ulsan.kr

\title{
The Timing of Femur Fracture Fixation is an Important Factor for Prolonged Mechanical Ventilation
}

\author{
Hyung Chul Choi, M.D. ${ }^{1}$, Kwang Hwan Jung, M.D., Ph.D. ${ }^{2}$, \\ Kyu Hyouck Kyoung, M.D., Ph.D. ${ }^{1,3}$, Seong Ho Choi, M.D. ${ }^{1,3}$ \\ ${ }^{1}$ Department of Surgery, Ulsan University Hospital, University of Ulsan College of Medicine, \\ Ulsan, Korea \\ ${ }^{2}$ Department of Orthopedic Surgery, Ulsan University Hospital, University of Ulsan College \\ of Medicine, Ulsan, Korea \\ ${ }^{3}$ Trauma Center, Division of Trauma Surgery, Department of Surgery, Ulsan University \\ Hospital, University of Ulsan College of Medicine, Ulsan, Korea
}

Purpose: Mechanical ventilation (MV) is an essential life-saving modality for severely injured patients. However, the long-term use of MV is a major risk factor for late mortality. The surgical correction of long bone fractures plays a critical role not only in improving functional outcomes, but also in reducing physiological derangements, including MV duration. This study investigated the factors affecting prolonged MV (PMV) in severely injured patients with femur fractures.

Methods: We retrospectively evaluated all severely injured patients (injury severity score $>15$ ) with femur fractures who were taken to the emergency department within 12 hours of the causative accidents between January 2016 and December 2018. PMV was defined as MV lasting for $\geq 7$ days. We analyzed the factors affecting PMV.

Results: In total, 35 patients were enrolled and 21 (33.3\%) were included in the PMV group. The PMV group required more red blood cell (RBC) transfusions within 7 days RBC (7dRBC) (12.8 vs. 6.8 units; $p=0.03$ ) and the time to femur fracture fixation (TFFF) was longer ( 7.9 vs. 2.7 days; $p=0.018$ ). The area under the curve (AUC) for TFFF was 0.740 ( $95 \%$ confidence interval [CI]: $0.572-0.908 ; p=0.018$ ) and the AUC for $7 \mathrm{dRBC}$ was 0.718 (95\% CI: 0.546-0.889; $p=0.031$ ).

Conclusions: This study indicates that TFFF is an independent risk factor for PMV. Early fixation of femur fractures might prevent PMV and its associated complications.

Keywords: Mechanical ventilation; Fracture of long bone; Fixation of femoral fracture 


\section{INTRODUCTION}

Since Trunkey described a trimodal distribution of trauma deaths in 1983 [1], this pattern has changed to a bimodal curve as a result of subsequent developments of trauma care systems [2,3]. Notwithstanding the improvements that have taken place in trauma deaths, the causes of death at various times elapsed from the injury have not changed. Late mortality has been reported to vary from $8 \%$ to $16.4 \%[3,4]$ and the major causes of trauma deaths remain the same; namely, sepsis and multiple organ dysfunction syndrome (MODS) attributable to the consequences of the initial insults, aggressive resuscitation, and treatment $[5,6]$. Mechanical ventilation (MV) is an essential life-saving modality for severely injured patients. However, the long-term use of MV is also a major risk factor, increasing the late mortality rate to $24.2-35.6 \%$ $[7,8]$.

A longer MV duration is correlated with a higher risk of pulmonary complications, and prolonged MV (PMV) increases the risk of mortality by 4.89 -fold [9]. The reported risk factors for PMV include a low Glasgow Coma Scale score, flail chest, a high abbreviated injury scale (AIS) score of the thorax, the amount of resuscitation fluid, and facial fracture $[10,11]$. Although numerous modalities have been proposed as ways to minimize complications, researchers have primarily focused on medical treatments. The surgical correction of long bone fractures also plays a critical role not only in improving functional outcomes, but also in reducing physiological derangements, including the duration of MV [12]. The timing of long bone fixation may be considered to be an important factor, as it is a physiological parameter that affects patients' clinical course and outcomes. Therefore, this study investigated the factors affecting PMV in severely injured patients with femur fractures.

\section{METHODS}

\section{Data collection and exclusion criteria}

The present study was approved by the Institutional Review Board, which waived informed consent because no additional intervention was performed (IRB No. 2019-
09-017). We retrospectively evaluated all severely injured patients (injury severity score [ISS] $>15$ ) with femur fractures who were taken to the emergency department within 12 hours of the causative accidents between January 2016 and December 2018.

We excluded patients aged $<18$ or $\geq 80$ years, those who died or were transferred to another hospital within 7 days, those who did not need MV, and those with femur fractures that did not need fixation due to amputation or lesions affecting areas such as the trochanter or condyle. To prevent bias from influencing the outcomes, we also excluded patients with end-stage renal disease on dialysis and those with critical injuries (AIS $\geq 5$ ) on any other part of the body.

\section{Measurement of variables}

The patients were divided into the following two groups based on MV duration: less than 7 days and 7 or more days, and PMV was defined as MV lasting for $\geq 7$ days. To analyze the factors affecting the MV duration, the following information was collected: age, sex, injury mechanism, revised trauma score (RTS), the AIS of all body regions, the amount of transfusion, and the time to femur fracture fixation (TFFF) after the accident.

\section{Statistical analysis}

Continuous variables were presented as mean \pm standard deviation and compared using the Mann-Whitney $U$ test. Categorical variables were presented as $\mathrm{n}(\%)$ and compared using the chi-square and Fisher exact tests. Variables with $p<0.05$ in the univariate analysis were included in the multivariable logistic regression analysis to identify predictors of PMV. We calculated the adjusted odds ratios (ORs) with 95\% confidence intervals (CIs) for independent predictors of PMV. All statistical tests were two-sided, with significance set to 0.05 . All statistical analyses were performed using SPSS for Windows, version 21.0 (IBM Corp., Armonk, NY, USA).

\section{RESULTS}

In total, 35 patients met the inclusion criteria (Fig. 1). All patients were injured by blunt mechanisms and 21 (60.0\%) 


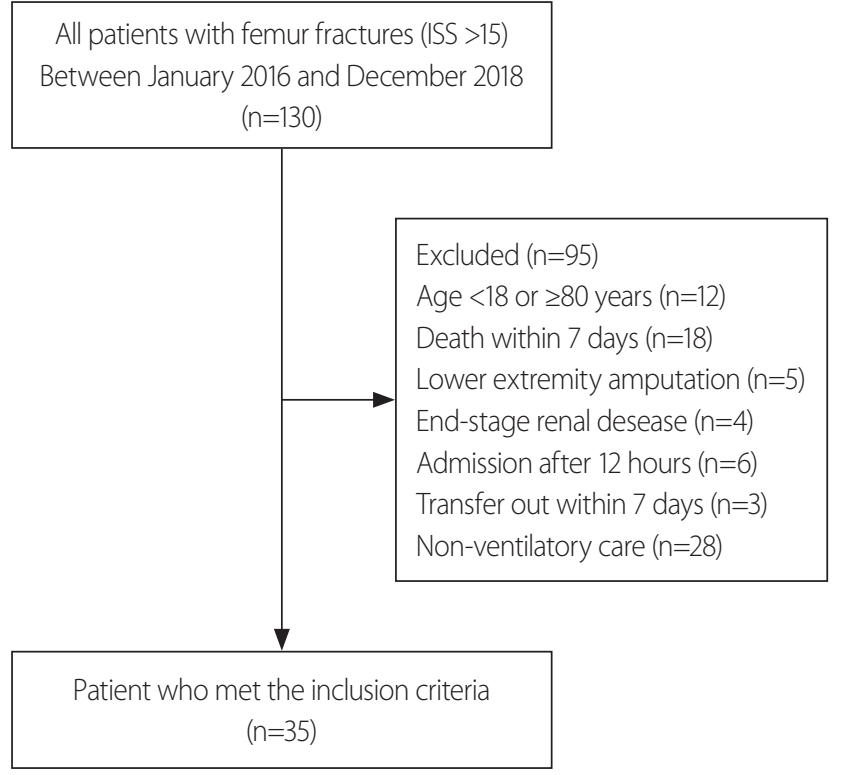

Fig. 1. Flow diagram showing patient enrollment. experienced PMV. There were no significant differences between the PMV and non-PMV groups in age, sex, timing of arrival to the trauma center, AIS, ISS, or emergency surgery. The PMV group had a higher RTS (10.4 \pm 1.6 vs. $11.5 \pm 0.9, p=0.022)$ and significantly more red blood cells (RBCs) transfused within 7 days (7dRBC) (6.8 \pm 2.5 vs. $12.8 \pm 9.1$ units, $p=0.03)$. The PMV group also had a longer TFFF ( $2.7 \pm 2.9$ vs. $7.9 \pm 11.2$ days, $p=0.018$ ). One patient in the PMV group died, but there was no significant difference in mortality between the groups (Table 1).

The receiver operating characteristic curves for RTS, TFFF, and 7dRBC showed significant predictive values. The area under the curve (AUC) of RTS was 0.716 (95\% CI: $0.110-0.458, p=0.033)$ and the AUC of $7 \mathrm{dRBC}$ was 0.718 (95\% CI: $0.546-0.889, p=0.031$ ). The AUC of TFFF was 0.740 (95\% CI: $0.572-0.908, p=0.018$ ); the cut-off value of 3.1 days had a sensitivity of $66.7 \%$ and specificity of

Table 1. Comparisons of demographic factors and clinical course according to the duration of mechanical ventilation

\begin{tabular}{|c|c|c|c|}
\hline Variable $(n=35)$ & Non-PMV & PMV & $p$-value \\
\hline Number & $14(40.0)$ & $21(60.0)$ & \\
\hline Age (years) & $56.2 \pm 17.3$ & $51.6 \pm 17.7$ & 0.490 \\
\hline Male & $12(85.7)$ & $20(95.2)$ & 0.551 \\
\hline Direct transport & $5(35.7)$ & $8(38.1)$ & 0.886 \\
\hline Scene to trauma center (minutes) & $180.4 \pm 164.1$ & $160.9 \pm 112.1$ & 0.946 \\
\hline Revised trauma score & $11.5 \pm 0.9$ & $10.4 \pm 1.6$ & 0.022 \\
\hline Injury severity score & $26.9 \pm 5.7$ & $26.81 \pm 7.3$ & 0.708 \\
\hline \multicolumn{4}{|l|}{ Abbreviated injury score } \\
\hline Head and neck & $1.3 \pm 1.6$ & $1.4 \pm 1.8$ & 0.909 \\
\hline Face & $0.6 \pm 0.9$ & $0.7 \pm 1.1$ & 0.936 \\
\hline Thorax and thoracic spine & $2.5 \pm 1.4$ & $2.2 \pm 1.5$ & 0.610 \\
\hline Abdomen and lumber spine & $1.4 \pm 1.6$ & $1.5 \pm 1.6$ & 0.842 \\
\hline Pelvis and extremities & $3.1 \pm 0.3$ & $3.1 \pm 0.3$ & 0.808 \\
\hline External & $0.4 \pm 0.5$ & $0.7 \pm 0.6$ & 0.198 \\
\hline Emergency surgery for CNS injury & $0(0)$ & $3(14.3)$ & 0.259 \\
\hline Emergency surgery for thoracoabdominal injury & $3(21.4)$ & $4(19.0)$ & 0.594 \\
\hline Time to femur fracture fixation (days) & $2.7 \pm 2.9$ & $7.9 \pm 11.2$ & 0.018 \\
\hline Ventilator days & $2.1 \pm 1.8$ & $20.8 \pm 23.7$ & $<0.001$ \\
\hline ICU days & $9.2 \pm 4.3$ & $30.9 \pm 23.8$ & $<0.001$ \\
\hline RBC transfusion within 7 days (units) & $6.8 \pm 2.5$ & $12.8 \pm 9.1$ & 0.030 \\
\hline Mortality & $0(0)$ & $1(4.8)$ & 1.000 \\
\hline
\end{tabular}

Values are presented as mean \pm standard deviation or number (\%).

PMV: prolonged mechanical ventilation, CNS: central nervous system, ICU: intensive care unit, RBC: red blood cell. 
78.6\% (Fig. 2).

To identify the risk factors, variables with $p<0.05$ in the univariate analysis were analyzed by multivariate logistic regression, and TFFF was categorized using the cut-off of 3 days derived from the above-presented results of this study. RTS (OR: 0.578, 95\% CI: 0.285-1.170, $p=0.128$ ) and the amount of $7 \mathrm{dRBC}$ (OR: 1.212, 95\% CI: 0.9871.490, $p=0.067$ ) did not reach statistical significance, but a TFFF $\geq 3$ days (OR: $11.873,95 \%$ CI: 1.644-85.767, $p=0.014$ ) was identified as a significant predictive factor for PMV (Table 2).

\section{DISCUSSION}

The numerous complications of multiple trauma are

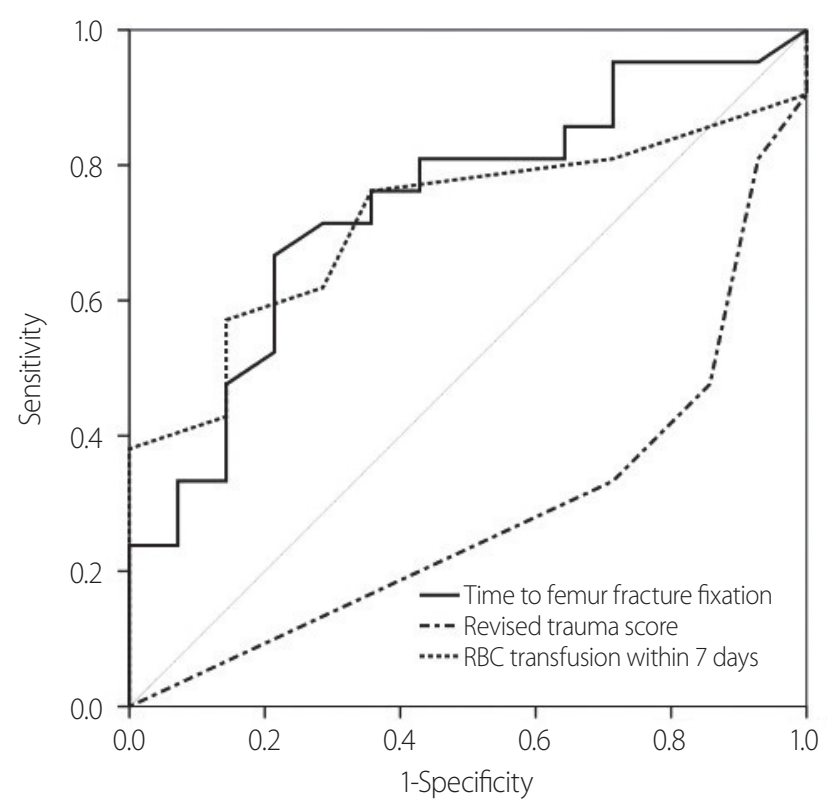

Fig. 2. Receiver operating characteristic curves of the risk factors for prolonged mechanical ventilation. related to the sequelae of resuscitation and life-saving procedures, in combination with the severity of the injury. Massive transfusion is associated with the lethal triad, electrolyte abnormalities, MODS, infection, and acute lung injury $[13,14]$. Though transfusion is a well-known major risk factor for MV and PMV [15,16], many trauma patients cannot avoid transfusion. In addition to efforts to decrease transfusions, therapeutic approaches should also be developed to reduce the duration of MV.

The timing of skeletal fixation remains controversial. Reconstructive secondary surgery is performed in survivors after damage control surgery or resuscitation. Secondary surgery promotes proinflammatory cytokines and aggravates organ dysfunction, imposing an additional burden due to the second-hit effect $[17,18]$. Contrary to emphasizing the risk of the second hit, recent studies have demonstrated the beneficial effects of early fixation, especially femur fracture. These studies reported that early stabilization of fractures reduced pulmonary complications, infections, hospital stay, and mortality [19-21]. Although the consensus on the advantages of early femur fixation has been widely accepted, the definition of "early" varies from within 12 hours to 4 days [17,20,21]. Furthermore, most studies did not explain the mechanisms by which early fixation decreases complications.

Moderate exercise upregulates antioxidants [22], and even passive physical activity improves levels of proinflammatory cytokines such as interleukin (IL)-6 and IL-10 [23]. Accordingly, although early mobilization is negatively associated with MV duration, it is problematic not to mobilize patients and even to change their position because of skeletal traction and concerns about additional injuries in patients with femur fractures [24]. Thus, early fixation of femur fractures followed by early mobilization may be more beneficial despite the risk of the second hit.

Pape et al. [25] reported an increased occurrence of

Table 2. Multivariate analysis of the risk factors for prolonged mechanical ventilation

\begin{tabular}{|c|c|c|c|}
\hline Variable $(n=35)$ & OR & $95 \% \mathrm{Cl}$ & $p$-value \\
\hline Revised trauma score & 0.578 & $0.285-1.170$ & 0.128 \\
\hline Time to femur fracture fixation, $\geq 3$ days & 11.873 & $1.644-85.767$ & 0.014 \\
\hline RBC transfusion within 7 days (units) & 1.212 & $0.987-1.490$ & 0.067 \\
\hline
\end{tabular}

OR: odds ratio, Cl: confidence interval, RBC: red blood cell. 
organ dysfunction when secondary surgery for major fractures was performed within less than 5 days, using IL-6 levels to assess the additional inflammatory burden caused by secondary surgery. They recommended that major skeletal surgery be delayed until after 5 days for safety [17]. Similarly to this, Cantu et al. [21] reported delaying surgery for femur fractures until at least 48 hours in all patients; moreover, severely injured patients (ISS $\geq 26$ ) also had higher mortality due to insufficient resuscitation when femur fracture fixation was performed within 12 hours. These differences can be interpreted in light of the clinical course of trauma. Proinflammatory cytokines are released immediately after injury and return to the normal range in about 5 days [25]. MODS develops concomitant with the course of systemic inflammation and peaks within 3-5 days [26]. Therefore, the better outcomes for early or delayed surgery result from avoiding the peak period of systemic inflammation. Although a detailed subgroup analysis was not performed in this study, the TFFF cut-off of 3.1 days for PMV seemed to result from the avoidance of peak systemic inflammation and organ dysfunction, combined with the effects of early mobilization.

The current study has some limitations. First, there was no correlation between PMV and ISS, which is one of the most important prognostic factors in trauma patients. We excluded patients with an AIS $\geq 5$ to eliminate bias caused by critical injuries to specific body regions; thus, the results might have been influenced by this narrow range of ISS. Second, we did not analyze specific conditions influencing MV or outcomes such as infection, delirium, and organ dysfunction. Despite these limitations, our findings suggest that early fixation of femur fractures might improve outcomes in patients with severe trauma.

\section{CONCLUSION}

This study indicates that TFFF is an independent risk factor for PMV. Early fixation of femur fractures might prevent PMV and its associated complications.

\section{REFERENCES}

1. Trunkey DD. Trauma. Accidental and intentional injuries account for more years of life lost in the U.S. than cancer and heart disease. Among the prescribed remedies are improved preventive efforts, speedier surgery and further research. Sci Am 1983;249:28-35.

2. Gunst M, Ghaemmaghami V, Gruszecki A, Urban J, Frankel H, Shafi S. Changing epidemiology of trauma deaths leads to a bimodal distribution. Proc (Bayl Univ Med Cent) 2010;23:349-54.

3. Kleber C, Giesecke M, Buschmann C. Overall distribution of trauma-related deaths in Berlin 2010: the weakest links of the chain of survival are emergency medicine and critical care: reply. World J Surg 2013;37:475.

4. Oyeniyi BT, Fox EE, Scerbo M, Tomasek JS, Wade CE, Holcomb JB. Trends in 1029 trauma deaths at a level 1 trauma center: impact of a bleeding control bundle of care. Injury 2017;48:5-12.

5. Kasotakis G, Sideris A, Yang Y, de Moya M, Alam H, King DR, et al. Aggressive early crystalloid resuscitation adversely affects outcomes in adult blunt trauma patients: an analysis of the Glue Grant database. J Trauma Acute Care Surg 2013;74:121521.

6. Jones DG, Nantais J, Rezende-Neto JB, Yazdani S, Vegas P, Rizoli S. Crystalloid resuscitation in trauma patients: deleterious effect of 5L or more in the first 24h. BMC Surg 2018;18:93.

7. Rubano JA, Paccione MF, Rutigliano DN, Vosswinkel JA, McCormack JE, Huang EC, et al. Outcomes following prolonged mechanical ventilation: analysis of a countywide trauma registry. J Trauma Acute Care Surg 2015;78:289-94.

8. Hough CL, Caldwell ES, Cox CE, Douglas IS, Kahn JM, White DB, et al. Development and validation of a mortality prediction model for patients receiving 14 days of mechanical ventilation. Crit Care Med 2015;43:2339-45.

9. Funk GC, Anders S, Breyer MK, Burghuber OC, Edelmann G, Heindl W, et al. Incidence and outcome of weaning from mechanical ventilation according to new categories. Eur Respir J 2010;35:88-94.

10. Okabe Y. Risk factors for prolonged mechanical ventilation in patients with severe multiple injuries and blunt chest trauma: a single center retrospective case-control study. Acute Med Surg 2018;5:166-72.

11. Agle SC, Kao LS, Moore FA, Gonzalez EA, Vercruysse GA, Todd SR. Early predictors of prolonged mechanical ventilation in major torso trauma patients who require resuscitation. Am J Surg 
2006;192:822-7.

12. Harvin JA, Harvin WH, Camp E, Caga-Anan Z, Burgess AR, Wade CE, et al. Early femur fracture fixation is associated with a reduction in pulmonary complications and hospital charges: a decade of experience with 1,376 diaphyseal femur fractures. J Trauma Acute Care Surg 2012;73:1442-8.

13. Sihler KC, Napolitano LM. Complications of massive transfusion. Chest 2010;137:209-20.

14. Wafaisade A, Lefering R, Bouillon B, Sakka SG, Thamm OC, Paffrath T, et al. Epidemiology and risk factors of sepsis after multiple trauma: an analysis of 29,829 patients from the trauma registry of the German Society for Trauma Surgery. Crit Care Med 2011;39:621-8.

15. Serpa Neto A, Juffermans NP, Hemmes SNT, Barbas CSV, Beiderlinden $\mathrm{M}$, Biehl $\mathrm{M}$, et al. Interaction between peri-operative blood transfusion, tidal volume, airway pressure and postoperative ARDS: an individual patient data meta-analysis. Ann Transl Med 2018;6:23.

16. Clendenen N, Weitzel N. Predictors of prolonged mechanical ventilation in adults after acute type-A aortic dissection repair-implications for the future. J Cardiothorac Vasc Anesth 2017;31:1562-63.

17. Pape HC, van Griensven M, Rice J, Gänsslen A, Hildebrand F, Zech S, et al. Major secondary surgery in blunt trauma patients and perioperative cytokine liberation: determination of the clinical relevance of biochemical markers. J Trauma 2001;50:9891000.

18. Pape HC, Schmidt RE, Rice J, van Griensven M, das Gupta R, Krettek C, et al. Biochemical changes after trauma and skeletal surgery of the lower extremity: quantification of the operative burden. Crit Care Med 2000;28:3441-8.

19. Byrne JP, Nathens AB, Gomez D, Pincus D, Jenkinson RJ. Timing of femoral shaft fracture fixation following major trauma: a retrospective cohort study of United States trauma centers. PLoS Med 2017;14:e1002336.

20. Nahm NJ, Como JJ, Wilber JH, Vallier HA. Early appropriate care: definitive stabilization of femoral fractures within 24 hours of injury is safe in most patients with multiple injuries. J Trauma 2011;71:175-85.

21. Cantu RV, Graves SC, Spratt KF. In-hospital mortality from femoral shaft fracture depends on the initial delay to fracture fixation and injury severity score: a retrospective cohort study from the NTDB 2002-2006. J Trauma Acute Care Surg 2014;76:1433-40.

22. Gomez-Cabrera MC, Domenech E, Viña J. Moderate exercise is an antioxidant: upregulation of antioxidant genes by training. Free Radic Biol Med 2008;44:126-31.

23. Winkelman C, Higgins PA, Chen YJ, Levine AD. Cytokines in chronically critically ill patients after activity and rest. Biol Res Nurs 2007;8:261-71.

24. Lai CC, Chou W, Chan KS, Cheng KC, Yuan KS, Chao CM, et al. Early mobilization reduces duration of mechanical ventilation and intensive care unit stay in patients with acute respiratory failure. Arch Phys Med Rehabil 2017;98:931-9.

25. Pape HC, Tsukamoto T, Kobbe P, Tarkin I, Katsoulis S, Peitzman A. Assessment of the clinical course with inflammatory parameters. Injury 2007;38:1358-64.

26. Dewar DC, Mackay P, Balogh Z. Epidemiology of post-injury multiple organ failure in an Australian trauma system. ANZ J Surg 2009;79:431-6. 